\title{
3 GHz, Yb-fiber laser based, few-cycle ultrafast source at the Ti:sapphire laser wavelength
}

\author{
Hung-Wen Chen, ${ }^{1, *}$ Haider Zia, ${ }^{2}$ JinKang Lim, ${ }^{1}$ Shanhui Xu, ${ }^{3}$ Zhongmin Yang, ${ }^{3}$ \\ Franz X. Kärtner, ${ }^{1,2,4}$ and Guoqing Chang ${ }^{1,4}$ \\ ${ }^{1}$ Research Laboratory of Electronics, Massachusetts Institute of Technology, 77 Mass Ave Cambridge MA 02139 \\ ${ }^{2}$ Center for Free-Electron Laser Science, DESY and University of Hamburg, Notkestraße 85, D-22607 Hamburg, Germany \\ ${ }^{3}$ State Key Laboratory of Luminescent Materials and Devices and Institute of Optical Communication Materials, \\ South China University of Technology, Guangzhou 510640, China \\ ${ }^{4}$ Physics Department, University of Hamburg, Luruper Chaussee 149, 22761 Hamburg, Germany \\ *Corresponding author: hungwen@mit.edu \\ Received Month X, 2013; revised Month X, XXXX; accepted Month X, XXXX; \\ posted Month X, XXXX (Doc. ID XXXXX); published Month X, XXXX
}

\begin{abstract}
We demonstrate a compact ultrafast source centered at $850 \mathrm{~nm}$ with $>200-\mathrm{nm}$ bandwidth (full width at half maximum) based on a 3-GHz Yb-fiber master-oscillator-power-amplifier system. The output pulses (with up to $13 \mathrm{~W}$ average power) from the laser system are coupled into short $(<50 \mathrm{~mm})$ pieces of photonic crystal fibers to excite broadband fiber-optic Cherenkov radiation; the resulting broad phase-matching bandwidth due to short fiber length produces up-converted spectrum spanning in the wavelength range of 750-950 $\mathrm{nm}$ with an average power of $94 \mathrm{~mW}, 184 \mathrm{~mW}$, and $380 \mathrm{~mW}$ for the fiber length of 28 $\mathrm{mm}, 37 \mathrm{~mm}$, and $48 \mathrm{~mm}$, respectively. The spectrum generated from the $37 \mathrm{~mm}$ fiber is then de-chirped by 8 double-chirped mirrors, leading to compressed pulses of $\sim 14 \mathrm{fs}$ in duration. Such an ultrafast source is a promising substitute of multi-GHz mode-locked Ti:sapphire lasers for applications in optical frequency metrology and multi-photon coherent microscopy.

(C) 2013 Optical Society of America
\end{abstract}

OCIS Codes: 060.2310, 060.5295, 140.7090, 190.4370, 320.2250, 320.7110.

Kerr-lens mode-locked Ti:sapphire lasers have become the workhorse in the field of ultrafast optics. Due to the remarkable gain bandwidth ranging from 650 to 1100 $\mathrm{nm}$, Ti:sapphire lasers with precise cavity-dispersion management emit optical pulses as short as 5 fs-the record short optical pulses ever directly generated from mode-locked lasers [1]. Besides countless time-domain applications utilizing the extremely short pulses, the wavelength range covered by Ti:sapphire lasers is of particular importance for many applications. For example, ultrafast Ti:sapphire lasers are the dominant light source in the field of nonlinear optical microscopy largely because optical pulses with wavelength around $800 \mathrm{~nm}$ are able to excite a wide range of important fluorophores via two-photon absorption [2].

Despite their extreme success, the lack of efficient and direct diode pumping has hampered the wide use of Ti:sapphire lasers outside research laboratories, which has spurred much research effort in developing alternative laser sources. One attractive candidate is diode-pumped mode-locked lasers based on $\mathrm{Cr}^{3+-}$ doped colquiriite crystals such as $\mathrm{Cr}^{3+}: \operatorname{LiSAF}[3]$, $\mathrm{Cr}^{3+}: \mathrm{LiSGaF}$ [4], and $\mathrm{Cr}^{3+}: \mathrm{LiCAF}$ [5]. These solid-state lasers provide broadly tunable ultrashort pulses around $800 \mathrm{~nm}$ with a duration as short as $\sim 10 \mathrm{fs}$ [6].

In this paper, we present another alternative derived from an ultrafast $\mathrm{Yb}$-fiber laser system. The state-of-theart $\mathrm{Yb}$-fiber oscillators are able to produce ultrashort pulses centered at $1.03 \mu \mathrm{m}$ with duration less than ten optical cycles after external compression $[7,8]$. To generate few-cycle pulses at the Ti:sapphire laser wavelength range, we perform frequency up-conversion using fiberoptic Cherenkov radiation (FOCR) inside a short piece of photonic crystal fiber (PCF). As ultrashort pulses propagate in a PCF with anomalous dispersion, they emit dispersive waves due to the high-order fiber dispersion - a phenomenon known as FOCR [9-11]. Depending on the sign of the third-order dispersion (TOD), the dispersive wave may locate in the wavelength region shorter (for positive TOD) or longer (for negative TOD) than the input-pulse's center wavelength [12]. FOCR has become a wavelength conversion technique [13-15], for example, to extend a Ti:sapphire laser's near-infrared output into the visible wavelength range with applications for biophotonics, carrier-envelope phase control of ultrashort pulses, and calibration of astrophysical spectrographs using high-precision frequency combs (known as astrocombs ), to name a few [16,17].

Despite originating from the $\chi^{(3)}$ nonlinear susceptibility of an optical fiber, FOCR shares a universal feature with other well-known $\chi^{(2)}$ nonlinear effects (e.g., second-harmonic generation): they all require phasematching to achieve efficient power conversion. In our recent study, we have introduced the concept of coherence length to quantify the FOCR bandwidth and its dependence on the PCF length $[18,19]$. Our experimental results using an octave-spanning Ti:sapphire laser as the driving source reveal that an optimum fiber length exists to maximize the FOCR bandwidth [19].

FOCR can be accurately modeled by the generalized nonlinear Schrödinger equation [20]. To be consistent with the following experimental results, we simulated an optical pulse centered at $1.045 \mu \mathrm{m}$ propagating inside PCF NL-3.2-945 available from NKT Photonics. The fiber exhibits zero-dispersion at $0.945 \mu \mathrm{m}$ with a mode field diameter of $2.8 \mu \mathrm{m}$ at $1.045 \mu \mathrm{m}$, corresponding to a fiber nonlinearity of $23 \mathrm{~W}^{-1} \mathrm{~km}^{-1}$. In the simulation, we fit the experimental dispersion curve provided by the 
manufacturer with a $12^{\text {th }}$ order polynomial. Figure 1(a) plots the spectrum evolution along the fiber length for an input Gaussian pulse with 110 fs full-width-halfmaximum (FWHM) and $780 \mathrm{pJ}$ energy. Beyond $2.5 \mathrm{~cm}$, the optical spectrum starts to rapidly extend to the shorter wavelength region; at $3.7-\mathrm{cm}$ fiber length (indicated by the dashed line), a broadband continuum forms at the shorter wavelength side reaching $700 \mathrm{~nm}$ in wavelength. Further propagation leads to an isolated and slowly blue-shifting FOCR, and its bandwidth becomes narrower due to a decreased phase-matching bandwidth for longer fiber length. Figure 1(b) shows another simulation with the fiber length fixed at $3.7 \mathrm{~cm}$ and the input pulse energy varied. The spectrum evolution in Fig. 1(b) follows a similar pattern as is in Fig. 1(a), and shows that a broadband FOCR develops for input pulse energy around $780 \mathrm{pJ}$ designated by the dashed line.
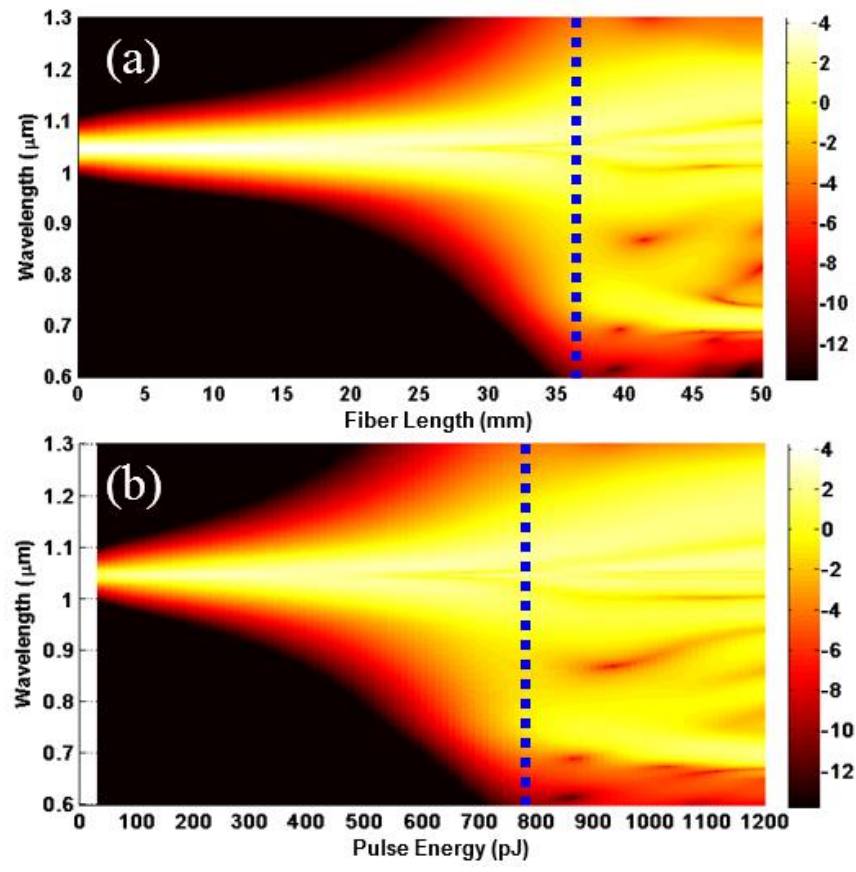

Fig. 1. (Color online) Simulation of FOCR by solving the generalized nonlinear Schrödinger equation. (a) FOCR evolution versus fiber length assuming $780 \mathrm{pJ}$ input pulse energy. The blue dashed line marks the $37-\mathrm{mm}$ fiber length where a broadband FOCR continuum forms. (b) FOCR evolution versus pulse energy for a PCF of $3.7 \mathrm{~cm}$ in length. The blue dashed line marks the 780-pJ pulse energy where a broadband FOCR continuum forms. The spectral intensity in both figures is presented on a logarithm scale.

Simulation results also show that for 110-fs input pulses, the FOCR accounts for $\sim 10 \%$ of the input pulse energy, resulting in a FOCR pulse of $\sim 100$ pJ. For a typical $100-\mathrm{MHz}$ repetition rate, the corresponding FOCR source produces an average power of $\sim 10 \mathrm{~mW}$, one order of magnitude less than can be typically achieved from a Ti:sapphire laser at the same repetition rate. To scale up the average power, here we derive our FOCR ultrafast source from a $3-\mathrm{GHz}$ Yb-fiber laser system, and demonstrate a $3-\mathrm{GHz}$ source at the Ti:sapphire laser wavelength with up to $380-\mathrm{mW}$ average power and >200 nm bandwidth (FWHM).

Figure 2 shows the schematic setup of the $3-\mathrm{GHz}$ FOCR laser source, mainly consisting of: 1) a $3-\mathrm{GHz}$ fundamentally mode-locked Yb-fiber oscillator [21], 2) a two-stage polarization-maintaining (PM) fiber amplifier to pre-amplify the oscillator pulses, 3) a diffraction-grating pair for pre-chirp management prior to further power amplification [22], 4) a double-clad Yb-fiber power amplifier, 5) a transmission-grating pair to compress the amplified pulses, 6) a short piece of PCF for wavelength conversion via FOCR, and 7) double-chirped mirrors $(\mathrm{DCMs})$ to compress the resulting pulses at $850 \mathrm{~nm}$.

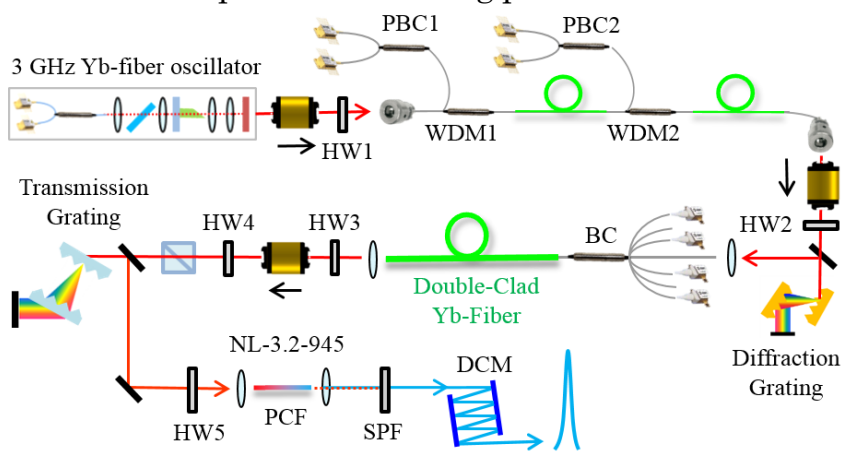

Fig. 2. (Color online) Schematic setup of the $3-\mathrm{GHz}$ ultrafast FOCR source. HW: half-wave plate; PBC: polarization beam combiner; WDM: wavelength-division multiplexing; BC: $(6+1)$ x 1 beam combiner; SPF: shortwavelength-pass optical filter that only transmits wavelength components below $950 \mathrm{~nm}$; and DCM: doublechirped mirror.

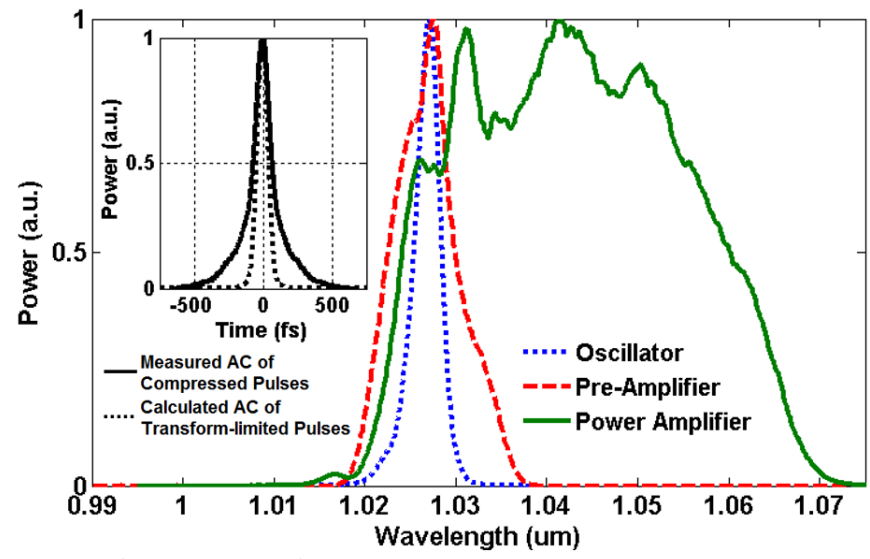

Fig. 3. (Color online) Optical spectra at the output of the 3$\mathrm{GHz}$ oscillator (blue dotted line), pre-amplifier (red dashed line), and power amplifier (green solid line), respectively. Inset: measured autocorrelation (AC) trace of the compressed pulses after the power-amplifier (solid line) and the calculated autocorrelation trace of the transformlimited pulse (dotted line).

The 3-GHz Yb-fiber oscillator, centered at $1027 \mathrm{~nm}$ with $3.5 \mathrm{~nm}$ bandwidth (blue dotted line in Fig. 3), produces 32 $\mathrm{mW}$ average power. The design and implementation of such a $3-\mathrm{GHz} \mathrm{Yb}$-fiber oscillator has been described in Ref. 21 . The output pulses are amplified to $1.05 \mathrm{~W}$ by a twostage pre-amplifier constructed from single-mode PM fibers; the resulting optical spectrum is broadened to $7 \mathrm{~nm}$ 
(red dashed line in Fig. 3). Before the pre-amplified pulses enter the power amplifier, we employ the pre-chirp management method demonstrated in Ref. 22 to achieve high-quality compressed pulses after the power amplifier. The pulses are pre-chirped by a diffraction-grating (600 line/mm) pair, and then amplified by a power amplifier constructed from 2.4-m double-clad PM Yb-fiber (10- $\mu \mathrm{m}$ core diameter with 0.08 numerical aperture) spliced to a PM $(6+1)$ x 1 beam combiner. The amplified pulses are spectrally broadened to $30-\mathrm{nm}$ bandwidth (green solid line in Fig. 3) during the amplification and are then compressed by a transmission-grating (1000 line/mm) pair. By properly pre-chirping the pre-amplified pulses, we achieve compressed pulses with $13-\mathrm{W}$ average power and the measured autocorrelation trace is $140 \mathrm{fs}$ in duration shown as the solid curve in the inset of Fig. 3. As a comparison, the autocorrelation trace of the transformlimited pulse calculated from the optical spectrum is plotted as the dotted curve, showing an autocorrelation duration of $95 \mathrm{fs}$.
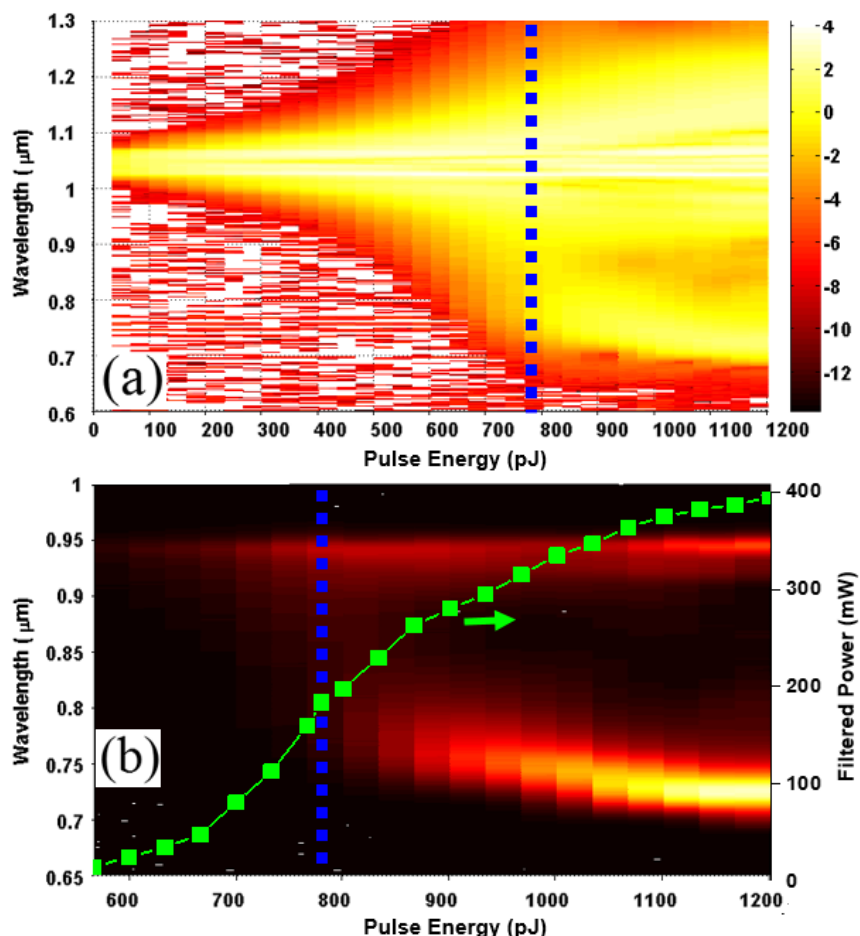

Fig. 4. (Color online) (a) Measured output spectra versus coupled pulse energy into 3.7-cm PCF NL-3.2-945. Spectral intensity is shown on a logarithm scale. (b) Measured output spectra after a short-wavelength-pass optical filter that blocks spectral components above $950 \mathrm{~nm}$. Spectral intensity is shown on a linear scale. The green curve in (b) shows the measured average power after the shortwavelength-pass optical filter. Blue dashed lines in both figures mark the $780 \mathrm{pJ}$ pulse energy corresponding to the broadband FOCR.

To compare with the simulation results, we couple the compressed pulses into 37-mm PCF NL-3.2-945. A halfwave plate (HW5 in Fig. 2) placed before the PCF mitigates the detrimental effect caused by the fiber's residual birefringence. Figure 4(a) records the measured spectra from the PCF output as a function of coupled pulse energy. A comparison between Fig. 4(a) and Fig. 1(a) indicates excellent agreement between simulation results and experimental measurements. As the simulation predicts, a broadband FOCR appears at the pulse energy of 780 pJ (marked by the dashed lines in Fig. 4), corresponding to $2.34-\mathrm{W}$ coupled average power. To clearly observe the evolution of FOCR spectra, we use a short-wavelength-pass optical filter to filter out the spectral components below $950 \mathrm{~nm}$, and plot them in Fig. 4(b), shown on a linear scale. It clearly shows that a spectral gap starts to form around $850 \mathrm{~nm}$ as pulse energy increases beyond $780 \mathrm{pJ}$, and an isolated FOCR spectrum appears with its center wavelength blue shifted with the increased pulse energy. The green curve marked with squares in Fig. 4(b) denotes the average power of the filtered spectrum, which increases monotonically for higher pulse energy. At 780-pJ pulse energy, 184-mW average power (i.e., 61-pJ pulse energy) is achieved.

The optimum pulse energy corresponding to the broadband, non-isolated FOCR depends on the PCF length: shorter PCF requires more input pulse energy. In addition to the 37-mm PCF, we have investigated another two fiber lengths and found that the required input pulse energy to achieve a broadband FOCR is about $440 \mathrm{pJ}$ for 48-mm PCF and $1430 \mathrm{pJ}$ for 28-mm PCF. Figure 5(a) plots the three broadband FOCR spectra after the shortwavelength-pass optical filter. Normalized to their peak at $940 \mathrm{~nm}$, the three spectra are of similar spectral shape centered around $850 \mathrm{~nm}$ with $>200-\mathrm{nm}$ bandwidth (FWHM). The average power of the filtered spectra are 94 $\mathrm{mW}, 184 \mathrm{~mW}$, and $380 \mathrm{~mW}$, respectively.
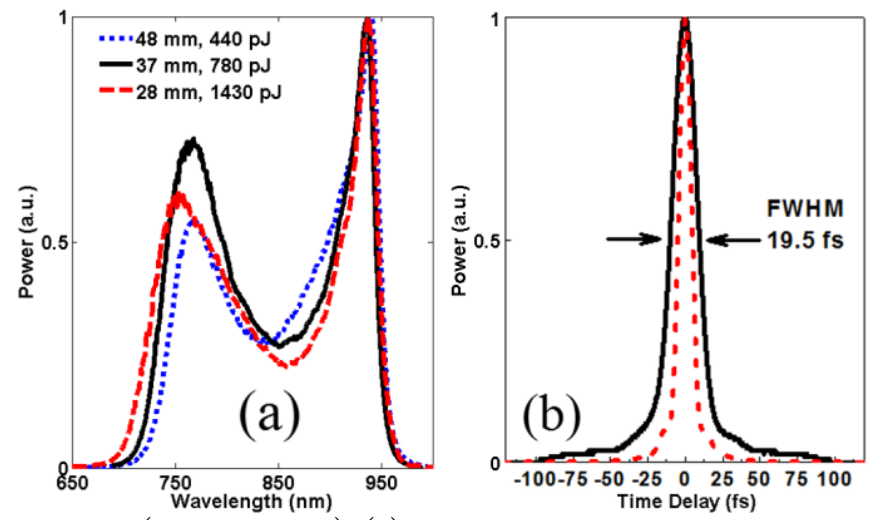

Fig. 5. (Color online) (a) Filtered spectra of broadband FOCR at three different fiber length: $28 \mathrm{~mm}$ (red dashed line), $37 \mathrm{~mm}$ (black solid line), and $48 \mathrm{~mm}$ (blue dotted line). These three spectra are generated at different input pulse energies: 1430 pJ (28-mm PCF), 780 pJ (37-mm $\mathrm{PCF})$, and $440 \mathrm{pJ}(48-\mathrm{mm} \mathrm{PCF})$. The spectra are all normalized to their spectral peak at $940 \mathrm{~nm}$. (b) Black solid line: measured autocorrelation trace of the compressed FOCR pulse using DCMs to compensate for the phase from the FOCR spectrum (i.e., black solid curve in (a)) generated from the 37-mm PCF with 780-pJ input pulse energy. Red dashed line: calculated autocorrelation trace of the transform-limited pulse given by the FOCR spectrum.

Since the FOCR is initiated by nonlinearity interacting with the higher-order dispersion, compressibility of such a broadband $(>200 \mathrm{~nm})$ FOCR spectrum remains in 
question. Since nearly the entire filtered spectrum falls into the PCF's normal dispersion region, we use homedesigned double-chirped mirrors (DCM) with negative group-delay dispersion to compensate for the spectral phase of the spectra in Fig. 5(a). The mirror comprises 96 alternating layers of $\mathrm{TiO}_{2}$ and $\mathrm{SiO}_{2}$, providing a groupdelay dispersion of $-70 \mathrm{fs}^{2}$. The black solid curve in Fig. 5(b) shows the measured shortest autocorrelation trace for the filtered FOCR spectrum achieved from 37- mm PCF and 780-pJ pulse energy. The autocorrelation trace after 8 DCMs has a duration (FWHM) of 19.5 fs. The total efficiency of the $8 \mathrm{DCMs}$ is $>99 \%$. Also shown in the same figure is the calculated autocorrelation trace (red dotted line) of the transform-limited pulse given by the corresponding FOCR spectrum (i.e., black solid curve in Fig. 5(a)). We estimate that the compressed pulses are $\sim 14$ fs in duration assuming a deconvolution factor of 1.4. We attribute the deviation from the 8-fs transform-limited duration to the uncompensated higher-order dispersion. Note that applying FOCR to Er-doped fiber lasers for broadband wavelength conversion followed by subsequent pulse compression has led to generation of 8 -fs pulses centered at $1.17 \mu \mathrm{m}[23]$.

In conclusion, we demonstrate that a combination of a high power $3-\mathrm{GHz} \mathrm{Yb}$-fiber laser system and FOCR provides new capabilities to ultrafast laser technology: 1) generation of $\sim 14 \mathrm{fs}$ pulses with smooth spectra and 2) broadband wavelength coverage overlapping with the Ti:sapphire lasers wavelength range. Despite the relatively low pulse energy of $\sim 100 \mathrm{pJ}$, such a multi-GHz few-cycle source at Ti:sapphire laser wavelength is of particular importance for applications in nonlinear biooptical imaging (e.g., two-photon fluorescence excitation microscopy) when photoinduced damage is caused by pulse energy rather than average power; increasing pulse repetition-rate while keeping the pulse energy below the damage threshold will improve signal-to-noise ratio and reduce data acquisition time [24]. In addition to the timedomain applications, stabilization of both the repetition rate and the carrier-envelope offset frequency of this source will lead to a frequency comb with $3-\mathrm{GHz}$ line spacing in the frequency domain. Such a frequency comb with multi-GHz line spacing at Ti:sapphire wavelength constitutes a critical device for precision calibration of astronomical spectrographs to search for exoplanets [25].

This work was supported under National Aeronautics and Space Administration (NASA) grant NNX10AE68G, the Air Force Office of Scientific Research grant (AFOSR) FA9550-12-1-0499, the Center for Free-Electron Laser Science, DESY Hamburg, and Masdar Institute of Science and Technology. The authors thank Duo Li for useful discussions.

\section{References}

1. R. Ell, U. Morgner, F. X. Kaertner, J. G. Fujimoto, E. P. Ippen, V. Scheuer, G. Angelow, T. Tschudi, M. J. Lederer, A. Boiko, and B. Luther-Davies, Opt. Lett. 26, 373-375 (2001).

2. B. E. D. M. I. of T. B. R. M. V. Scientist and P. S. P. of M. and B. E. M. I. of Technology, Handbook of
Biomedical Nonlinear Optical Microscopy (Oxford University Press, 2008).

3. U. Demirbas, M. Schmalz, B. Sumpf, G. Erbert, G. S. Petrich, L. A. Kolodziejski, J. G. Fujimoto, F. X. Kärtner, and A. Leitenstorfer, Opt. Express 19, 20444 20461 (2011).

4. U. Demirbas, D. Li, J. R. Birge, A. Sennaroglu, G. S. Petrich, L. A. Kolodziejski, F. X. Kaertner, and J. G. Fujimoto, Opt. Express 17, 14374-14388 (2009).

5. U. Demirbas, R. Uecker, D. Klimm, and J. Wang, Appl. Opt. 51, 8440-8448 (2012).

6. S. Uemura and K. Torizuka, Opt. Lett. 24, 780-782 (1999).

7. A. Chong, H. Liu, B. Nie, B. G. Bale, S. Wabnitz, W. H. Renninger, M. Dantus, and F. W. Wise, Opt. Express 20, 14213-14220 (2012).

8. Y. Lan, Y. Song, M. Hu, B. Liu, L. Chai, and C. Wang, Opt. Lett. 38, 1292-1294 (2013).

9. P. K. A. Wai, C. R. Menyuk, Y. C. Lee, and H. H. Chen, Opt. Lett. 11, 464-466 (1986).

10. V. I. Karpman, Phys. Rev. E 47, 2073-2082 (1993).

11. N. Akhmediev and M. Karlsson, Phys. Rev. A 51, 2602-2607 (1995).

12. D. V. Skryabin, F. Luan, J. C. Knight, and P. St. J. Russell, Science 301, 1705 (2003).

13. L. Tartara, I. Cristiani, and V. Degiorgio, Appl Phys B 77, 307-311 (2003).

14. X. Liu, G. E. Villanueva, J. Laegsgaard, U. Moller, H. Tu, S. A. Boppart, and D. Turchinovich, IEEE Photonics Technology Letters 25, 892-895 (2013).

15. H. Tu and S. A. Boppart, Opt. Express 17, 9858-9872 (2009).

16. Y. Deng, F. Lu, and W. Knox, Opt. Express 13, 45894593 (2005).

17. G. Chang, C.-H. Li, A. Glenday, G. Furesz, N. Langellier, L.-J. Chen, M. W. Webber, J. Lim, H.-W. Chen, D. F. Phillips, A. Szentgyorgyi, R. L. Walsworth, and F. X. Kaertner, in Conference on Lasers and Electro-Optics 2012, OSA Technical Digest (online) (Optical Society of America, 2012), p. CF2C.4.

18. G. Chang, L.-J. Chen, and F. X. Kärtner, Opt. Lett. 35, 2361-2363 (2010).

19. G. Chang, L.-J. Chen, and F. X. Kärtner, Opt. Express 19, 6635-6647 (2011).

20. G. P. Agrawal, Nonlinear Fiber Optics (Academic Press, San Diego, 2001), $3^{\text {rd }}$ ed.

21. H.-W. Chen, G. Chang, S. Xu, Z. Yang, and F. X. Kärtner, Opt. Lett. 37, 3522 (2012).

22. H.-W. Chen, J. Lim, S.-W. Huang, D. N. Schimpf, F. X. Kärtner, and G. Chang, Opt. Express 20, 28672 (2012).

23. A. Sell, G. Krauss, R. Scheu, R. Huber, and A. Leitenstorfer, Opt. Express 17, 1070 (2009).

24. N. Ji, J. C. Magee, and E. Betzig, Nature Methods 5, 197 (2008).

25. C.-H. Li, A. J. Benedick, P. Fendel, A. G. Glenday, F. X. Kartner, D. F. Phillips, D. Sasselov, A. Szentgyorgyi, and R. L. Walsworth, Nature 452, 610 (2008). 


\section{References}

1. R. Ell, U. Morgner, F. X. Kaertner, J. G. Fujimoto, E. P. Ippen, V. Scheuer, G. Angelow, T. Tschudi, M. J. Lederer, A. Boiko, and B. Luther-Davies, "Generation of 5-fs pulses and octave-spanning spectra directly from a Ti:sapphire laser," Opt. Lett. 26, 373-375 (2001).

2. B. E. D. M. I. of T. B. R. M. V. Scientist and P. S. P. of M. and B. E. M. I. of Technology, Handbook of Biomedical Nonlinear Optical Microscopy (Oxford University Press, 2008).

3. U. Demirbas, M. Schmalz, B. Sumpf, G. Erbert, G. S. Petrich, L. A. Kolodziejski, J. G. Fujimoto, F. X. Kärtner, and A. Leitenstorfer, "Femtosecond Cr:LiSAF and $\mathrm{Cr}$ :LiCAF lasers pumped by tapered diode lasers," Opt. Express 19, 20444-20461 (2011).

4. U. Demirbas, D. Li, J. R. Birge, A. Sennaroglu, G. S. Petrich, L. A. Kolodziejski, F. X. Kaertner, and J. G. Fujimoto, "Low-cost, single-mode diode-pumped Cr:Colquiriite lasers," Opt. Express 17, 14374-14388 (2009).

5. U. Demirbas, R. Uecker, D. Klimm, and J. Wang, "Low-cost, broadly tunable (375-433 nm \& 746$887 \mathrm{~nm}$ ) Cr:LiCAF laser pumped by one single-spatialmode diode," Appl. Opt. 51, 8440-8448 (2012).

6. S. Uemura and K. Torizuka, "Generation of 12-fs pulses from a diode-pumped Kerr-lens mode-locked Cr:LiSAF laser," Opt. Lett. 24, 780-782 (1999).

7. A. Chong, H. Liu, B. Nie, B. G. Bale, S. Wabnitz, W. H. Renninger, M. Dantus, and F. W. Wise, "Pulse generation without gain-bandwidth limitation in a laser with self-similar evolution," Opt. Express 20, 1421314220 (2012).

8. Y. Lan, Y. Song, M. Hu, B. Liu, L. Chai, and C. Wang, "Enhanced spectral breathing for sub-25 fs pulse generation in a Yb-fiber laser," Opt. Lett. 38, 12921294 (2013).

9. P. K. A. Wai, C. R. Menyuk, Y. C. Lee, and H. H. Chen, "Nonlinear pulse propagation in the neighborhood of the zero-dispersion wavelength of monomode optical fibers," Opt. Lett. 11, 464-466 (1986).

10. V. I. Karpman, "Radiation by solitons due to higherorder dispersion," Phys. Rev. E 47, 2073-2082 (1993).

11. N. Akhmediev and M. Karlsson, "Cherenkov radiation emitted by solitons in optical fibers," Phys. Rev. A 51, 2602-2607 (1995).

12. D. V. Skryabin, F. Luan, J. C. Knight, and P. St. J. Russell, "Soliton self-frequency shift cancellation in photonic crystal fibers," Science 301, 1705 (2003).

13. L. Tartara, I. Cristiani, and V. Degiorgio, "Blue light and infrared continuum generation by soliton fission in a microstructured fiber," Appl Phys B 77, 307-311 (2003).

14. X. Liu, G. E. Villanueva, J. Laegsgaard, U. Moller, H. Tu, S. A. Boppart, and D. Turchinovich, "Low-Noise Operation of All-Fiber Femtosecond Cherenkov Laser," IEEE Photonics Technology Letters 25, 892895 (2013).
15. H. Tu and S. A. Boppart, "Optical frequency upconversion by supercontinuum-free widely-tunable fiber-optic Cherenkov radiation," Opt. Express 17, 9858-9872 (2009).

16. Y. Deng, F. Lu, and W. Knox, "Fiber-laser-based difference frequency generation scheme for carrierenvelope-offset phase stabilization applications," Opt. Express 13, 4589-4593 (2005).

17. G. Chang, C.-H. Li, A. Glenday, G. Furesz, N. Langellier, L.-J. Chen, M. W. Webber, J. Lim, H.-W. Chen, D. F. Phillips, A. Szentgyorgyi, R. L. Walsworth, and F. X. Kaertner, "Spectrally flat, broadband visiblewavelength astro-comb," in Conference on Lasers and Electro-Optics 2012, OSA Technical Digest (online) (Optical Society of America, 2012), p. CF2C.4.

18. G. Chang, L.-J. Chen, and F. X. Kärtner, "Highly efficient Cherenkov radiation in photonic crystal fibers for broadband visible wavelength generation," Opt. Lett. 35, 2361-2363 (2010).

19. G. Chang, L.-J. Chen, and F. X. Kärtner, "Fiber-optic Cherenkov radiation in the few-cycle regime," Opt. Express 19, 6635-6647 (2011).

20. G. P. Agrawal, Nonlinear Fiber Optics (Academic Press, San Diego, 2001), $3^{\text {rd }}$ ed.

21. H.-W. Chen, G. Chang, S. Xu, Z. Yang, and F. X. Kärtner, "3 GHz, fundamentally mode-locked, femtosecond Yb-fiber laser," Opt. Lett. 37, 3522-3524 (2012).

22. H.-W. Chen, J. Lim, S.-W. Huang, D. N. Schimpf, F. X. Kärtner, and G. Chang, "Optimization of femtosecond Yb-doped fiber amplifiers for highquality pulse compression," Opt. Express 20, 28672 (2012).

23. A. Sell, G. Krauss, R. Scheu, R. Huber, and A. Leitenstorfer, "8-fs pulses from a compact Er:fiber system: quantitative modeling and experimental implementation," Optics Express 17, 1070 (2009).

24. N. Ji, J. C. Magee, and E. Betzig, "High-speed, lowphotodamage nonlinear imaging using passive pulse splitters," Nature Methods 5, 197 (2008).

25. C. Li, A. J. Benedick, P. Fendel, A. G. Glenday, F. X. Kärtner, D. F. Phillips, D. Sasselov, A. Szentgyorgyi, and R. L. Walsworth, "A laser frequency comb that enables radial velocity measurements with a precision of $1 \mathrm{~cm} \mathrm{~s}^{-1}$," Nature 452, 610-612 (2008). 